\title{
An algorithmic definition of the axial map
}

\author{
Alasdair Turner, Alan Penn, Bill Hillier \\ The Bartlett, University College London, 1-19 Torrington Place, London WC1E 6BT, England; \\ e-mail: a.turner@ucl.ac.uk, a.penn@ucl.ac.uk, b.hillier@ucl.ac.uk \\ Received 27 November 2003; in revised form 27 August 2004
}

\begin{abstract}
The fewest-line axial map, often simply referred to as the 'axial map', is one of the primary tools of space syntax. Its natural language definition has allowed researchers to draw consistent maps that present a concise description of architectural space; it has been established that graph measures obtained from the map are useful for the analysis of pedestrian movement patterns and activities related to such movement: for example, the location of services or of crime. However, the definition has proved difficult to translate into formal language by mathematicians and algorithmic implementers alike. This has meant that space syntax has been criticised for a lack of rigour in the definition of one of its fundamental representations. Here we clarify the original definition of the fewest-line axial map and show that it can be implemented algorithmically. We show that the original definition leads to maps similar to those currently drawn by hand, and we demonstrate that the differences between the two may be accounted for in terms of the detail of the algorithm used. We propose that the analytical power of the axial map in empirical studies derives from the efficient representation of key properties of the spatial configuration that it captures.
\end{abstract}

\section{Introduction}

Our aim in this paper is to demonstrate an algorithm for the construction of an axial map of architectural space (Hillier and Hanson, 1984; Hillier et al, 1983). The map comprises a set of lines drawn through the 'space' of a two-dimensional plan of a building or urban area (see figure 1). In use, the map is connected into a graph so that the lines are represented as nodes, and the intersections of lines as connections between the nodes. As such, it is very similar to numerous graph approaches that attempt to quantify the configuration of space, dating from at least March and Steadman (1971), who examined the relationship between rooms in buildings, and Krüger (1979)

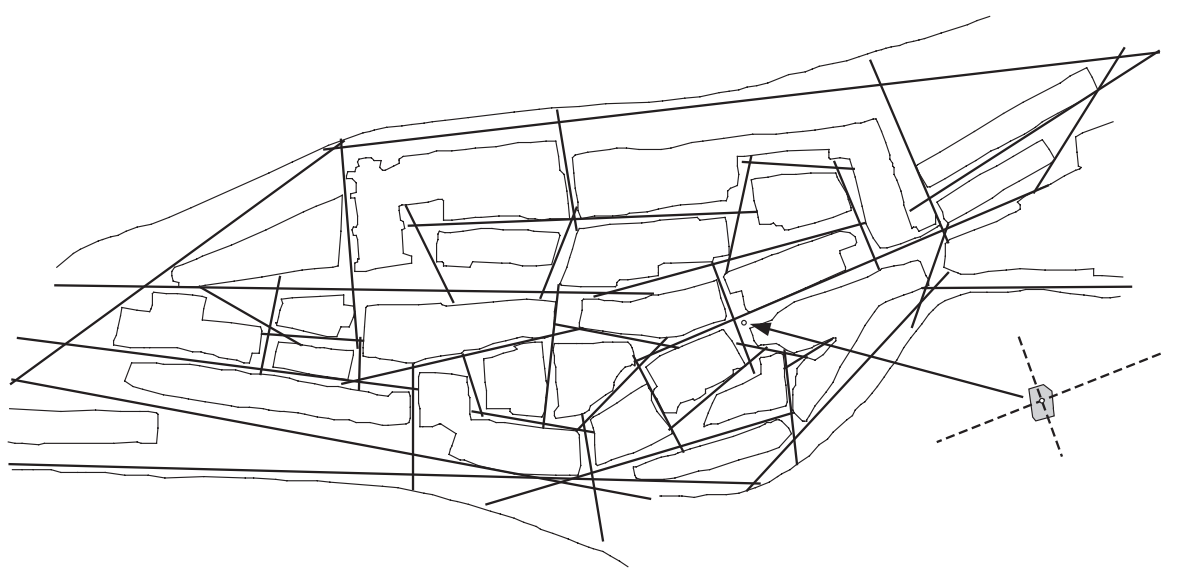

Figure 1. The original hand-drawn axial map of Gassin, France, vectorised from figure 28 in Hillier and Hanson (1984, page 91), with detail of 'stringy' (axial) and 'beady' (convex) extensions of a point, after figure 27. 
who examined the relationship of whole buildings in urban plans. Although these methods, and later ones (including others introduced by Hillier and Hanson themselves) are similar in that they construct a graph of spatial components, it is the axial map that has captured the imagination of many architects, designers, and urban planners, and become the mainstay of space syntax tools. Graph measures obtained from axial maps have been used to analyse successfully the effect of configuration of space on pedestrian movement in urban areas (Hillier et al, 1993; Peponis et al, 1989), traffic flows (Penn et al, 1998), crime distribution (Hillier and Shu, 2000), and land values (Desyllas, 2000), amongst many others (see de Holanda, 1999; Hanson, 2003; Peponis et al, 2001; UCL, 1997). Extensive research into axial maps has also led to their considerable usage in architectural and planning practice in the United Kingdom (and also elsewhere), particularly related to pedestrianisation as, for example, in the recent remodelling of London's Trafalgar Square (Space Syntax Limited, 2003).

The axial map was introduced after observation of real systems and experimentation with generative algorithms. Hillier and Hanson (1984) noted that urban space in particular seems to comprise two fundamental elements - 'stringiness' and 'beadiness' such that the space of the systems tends to resemble beads on a string (see inset in figure 1). They write:

"We can define 'stringiness' as being to do with the extension of space in one dimension, whereas 'beadiness' is to do with the extension of space in two dimensions" (page 91).

Hence, the epistemology of their methodology involves the investigation of how space is constructed in terms of configurations of interconnected beads and strings. To this end, they develop a more formal definition of the elements in which strings become 'axial lines' and beads 'convex spaces'. The definition they give is one that is easily understood by human researchers, but which, it has transpired, is difficult to translate into a computational approach:

"An axial map of the open space structure of the settlement will be the least set of [axial] lines which pass through each convex space and makes all axial links" (Hillier and Hanson, 1984, pages $91-92$ ).

The term axial line is defined as the longest line ${ }^{(1)}$ that can be drawn through an arbitrary point in the spatial configuration (see inset figure 1). Similarly, the term convex space is a 'fully fat' convex polygon around a point (see inset figure 1). To 'make all axial links' is to ensure that all axial lines are connected together if they possibly can be. However, it has recently been pointed out that, for a computational implementation, this apparently rigorous definition contains a problem (Batty and Rana, 2004; Ratti, 2004): the set of axial lines that fulfil these criteria cannot be precisely defined.

Hillier and Hanson have, in fact, become victim to their own precision. As a researcher recently remarked online: "do geographers spend so long defining road centre lines [as the space syntax community spends debating axial lines]?" The answer is, of course not. The map of the open space is a cartographer's artefact, and includes only features that he or she considers important. The basis for a road-centre line is that it should simply follow the centre of the road. If one then asks questions such as 'at exactly what point is a road considered a road and not a path?' we descend into an ultimately pointless debate. Batty and Rana (2004) sidestep this debate, and suggest that the definition of an axial line be broadened to include a range of differently specified sets of lines to be studied for their own interest. However, this approach

(1) Sometimes the 'longest visibility line' is referred to; however, the axial line as Hillier and Hanson define it is a purely geometrical entity. 
ignores the original purpose of the axial lines: to represent the 'stringy' connections between 'beads' of space. The stringy connections might as well be road-centre lines or any other topologically connecting form. For example, Thomas and Donikian (2000) suggest the connection of open space via a combination of Voronoï diagrams (to cover the 'beads') and sparse visibility graphs (to cover the 'strings') in order to model pedestrian movement patterns, or Jiang and Claramunt (2002) and Cutini et al (2004) suggest axial-like maps derived from sparse visibility graphs between points at key intersections and changes of direction. Given these two considerations - one, that the eventual definition must necessarily be arbitrary and open to interpretation, and two, that there are countless ways one might connect together stringy space-why pursue the original definition of the axial map further? The answer is that the results of the technique have been thoroughly investigated over the last twenty years. As just a single example, Carvalho and Penn (2004) compare thirty-six axial maps of cities (each comprising tens of thousands of hand-drawn axial lines), in order to show that there is a scale invariance in a range of line lengths exhibited in each. It is important to show that these lines may be defined consistently, if only for results such as these to have any weight.

In this paper, we first review the problem, and then restate Hillier and Hanson's definition in a more rigorous form. We describe previous approaches to the generation of axial maps in the light of this definition. We then demonstrate that it is possible to generate precise axial maps according to the definition, but subject to a stricter definition of lines that may be eligible to be considered axial lines. Our solution is intimately intertwined with a previous solution by Peponis et al (1998), and our method generalises their solution to a generic arbitrary map of open space. Our technique, though, varies in two ways. First, and crucially, perhaps, it is guaranteed to give a graph-unique minimal set of axial lines ('graph-unique' means unique in terms of the graph formed from the axial lines, not always the actual lines themselves). Second, our algorithm works for detailed plans of urban system, whereas, in general, Peponis et al's algorithm may be applied only to much simplified maps of the open space, because of implementational details of their method which we articulate below. After we have constructed our solution, we include a discussion of the results of applying a computersoftware implementation of our algorithm to three test cases. First, the 'original' axial map of the village of Gassin [shown in figure 1 and reproduced from Hillier and Hanson (1984)] has become a test case for axial-map generation. We compare our results on Gassin with those presented by Peponis et al. We also examine two further cases to demonstrate the applicability of the algorithm: a large public building (the National Gallery in London), and an urban area studied by Hillier and Hanson (the Barnsbury area of London).

\section{Background}

\subsection{The problem}

There are two aspects to the computational problem with Hillier and Hanson's (1984) definition given in section 1 above. One, there is indeed a problem, and it stems from a famous problem posed by Klee in 1973: how many guards does it take to fully surveil an art gallery? (O'Rourke, 1987). Two, there is a confusion which pervades the critique of the axial map. The confusion appears to stem from the proximity in Hillier and Hanson's text of the definition of a convex map. A convex map is the minimal set of convex spaces within the configuration. The natural language definition of a convex space which they give is not that strict; for our purposes, their 'fully fat' space is most easily regarded as the maximal convex polygon around a point (that is, the largest convex polygon that can be formed about a point, see figure 2, over). Batty and Rana (2004) 


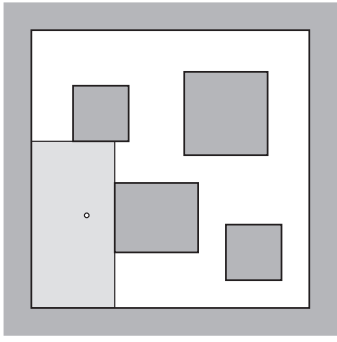

(a)

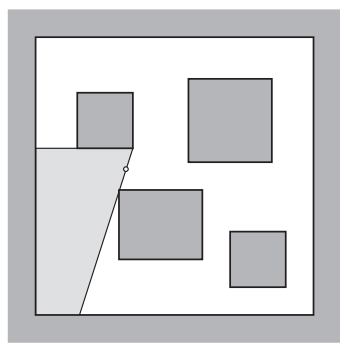

(b)

Figure 2. (a) A convex space may be regarded as the maximal convex polygon about a point. (b) In general there are an infinite number of possible maximal convex polygons, as the precise location of their edges depends on the positioning of the associated point.

point out that there is, in general, no algorithmic way to calculate the minimal set of maximal convex polygons, and hence no way to form a convex map. This is a problem for the definition of Hillier and Hanson's (1984) convex map; however, it is not the precise problem that afflicts the generation of an axial map (although it is closely related to it). Batty and Rana, as others, follow the implication that the lines in an axial map must 'pass through' (in some way) all the convex spaces in the convex map. In fact, Hillier and Hanson, do not phrase it in quite that manner: they simply state that the lines in an axial map must pass through 'each convex space' - that is, every convex space, not simply the minimal set. This might at first seem a more difficult condition to fulfill, and the phrase 'pass through' is, of course, imprecise in itself, but we can rephrase it in a few steps to form a simpler description of the condition. We start with the condition that the set of axial lines, taken together, should cross into every possible maximal convex polygon. In other words, the lines should pass through every region that is entirely intervisible (as all points in a convex polygon are necessarily intervisible). In other words again, the lines should pass through every region which is not surveillable from the rest of the plan (otherwise, of course, it would be intervisible with the region). That is, finally in this chain of argument, the lines should surveil the plan fully.

This statement forms the basis of the definition of the axial map. Stated explicitly:

Definition: An axial map is the minimal set of axial lines ${ }^{(2)}$ such that the set taken together fully surveils the system, and that every axial line that may connect two otherwise-unconnected lines is included.

The second half of this definition arises from the condition that all axial links should be made. The first half of the definition leads to the real problem: which lines form the minimal set that can fully surveil an arbitrary plan? In the language of mathematics, the plan becomes 'an arbitrary polygon with holes'. That is, a polygonal system boundary of some sort (the polygon) with numerous polygonal internal partitions, or buildings (the holes). The problem is a special case of what is known as the 'guardplacement problem': that is, where should guards be placed in order to surveil the system fully? Although we can know the upper limit on the number of guards necessary to surveil the system (Bjorling-Sachs and Souvaine, 1995; Hoffman et al, 1991), it is, in general, impossible to find where to put them. The problem is not intractable, but it is NP-hard (Lee and Lin, 1986), which translates roughly to 'solvable, but not solvable in polynomial time' which, in turn, means that, as the number of holes or the complexity of the polygonal edges rises, the time it takes to solve the problem rises

(2) The definition of an axial line follows Penn et al (1997); see section 2.2. 
exponentially until, like the losing gambler who doubles his or her stake each time, our computer runs out of resources. In order to solve the problem, the axial line must be further specified. In addition, previous authors have still had to approximate the minimal set of lines that fulfil this condition, as they have started with the first half of the definition rather than the second.

\subsection{All-line axial map reduction}

Solutions to the generation of axial maps have existed since 1986, when Czapski and Penn wrote a script for a now defunct computer-aided design package. However, in the literature the first real attempt at a solution is by Peponis et al (1998). Before attempting a solution, they first simplified the problem: rather than trying to find a minimal set of axial lines from the set of all possible axial lines (necessarily infinite, as there are infinite points to generate them within the space), they start with a subset of the lines first published by Penn et al (1997). Penn et al redefined an axial line as any line that joins two intervisible vertices within the system in one of the following ways: (a) both intervisible vertices are convex; or (b) one is convex and one is reflex, and the line joining the vertices can be and is extended through open space past the reflex vertex; or (c) both are reflex, and the line joining the vertices can be and is extended through open space past both vertices. The three types of possible axial line are shown in figure 3. The set of all possible such lines is called an 'all-line map' (Penn et al, 1997). Figure 4 shows detail for an all-line axial map generated for Gassin. Obviously, the number of lines generated for an all-line axial map varies with number of vertices in the polygonal boundary, and so the map changes as the level of detail is increased. The polygonal boundary and holes for the map shown in figures 1 and 4 comprises 1080 edge segments, which yields 5217 axial lines in the all-line map.

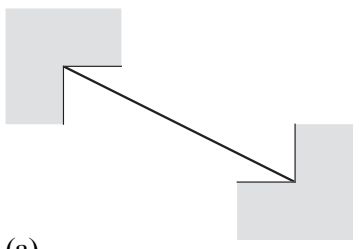

(a)

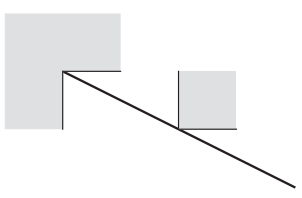

(b)

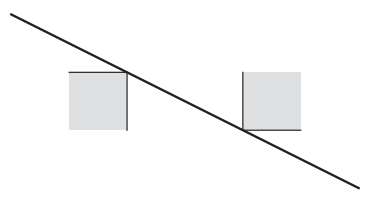

(c)

Figure 3. Possible types of axial line as defined by Penn et al (1997): (a) convex-convex vertex, (b) convex - reflex vertex, (c) reflex - reflex vertex.

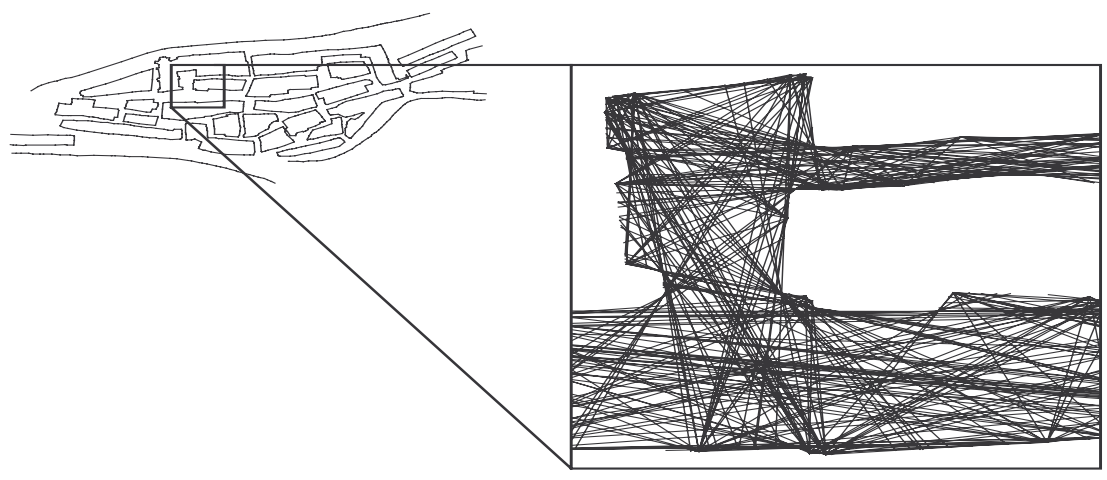

Figure 4. Detail of the all-line map generated from a vectorised version of the original Gassin map shown in figure 1. 


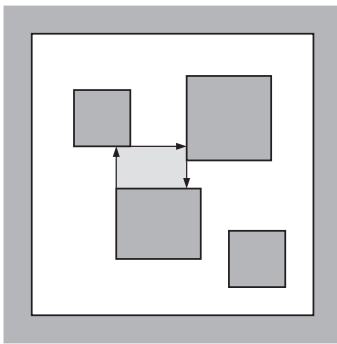

(a)

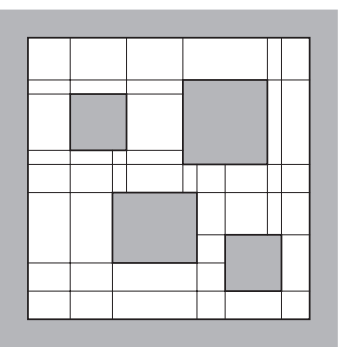

(b)

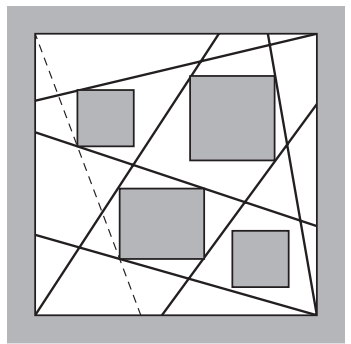

(c)

Figure 5. (a) The formation of an overlapping convex space within a configuration using s-lines; (b) all s-lines for the system; (c) the fewest-line map as generated with Peponis's preliminary algorithm, which does not find the dotted line.

Having left themselves with a countable set of lines, Peponis et al then attempted to minimise this number of lines to a fewest-line set. They were careful to avoid the use of the term 'axial line', and instead called them m-lines as they realised that their formalisation does not necessarily lead to the axial map described by Hillier and Hanson. They first formed overlapping convex spaces by extending the faces of the polygonal boundary and holes across open space until they hit another object, as shown in figure 5(a) (they called these extensions s-lines). In order to reduce the number of axial lines to a minimal set, they employed a well known method used to tackle an NP-hard problem - a greedy algorithm. A greedy algorithm works by first selecting a line which has the maximum value for some criteria, then taking the line with the second highest value of unmet criteria, and so until all the criteria are covered. Thus, the axial line which crosses the maximum number of s-lines is selected. The selected line forms one of the fewest set. Next, the line crossing the most overlapping convex spaces or s-lines (not crossed by the first axial line) is selected, and added to the set. An so on, until no more overlapping convex spaces or s-lines remain. The result can be impressive, as shown in figure 5(c). What is more, Peponis et al's algorithm is guaranteed to find a set of lines that surveil a system, as the s-lines represent the change between being able to see a face and not being able to see a face. However, although impressive, the algorithms do not quite reproduce what is drawn by hand. The dotted line shown in figure 5(c) is omitted by this first algorithm advanced by Peponis et al. In order to include this 'missing' line, Peponis et al followed a line of reasoning implied by Hillier and Hanson (1984). They argued that, because axial lines are primarily a device to describe possible lines of movement (that is, the stringiness we refer to above), handdrawn maps always complete 'nontrivial circulation loops'. A nontrivial circulation loop is a topological ring drawn around every hole in the system. Thus, Peponis et al proposed a method to ensure that every topological ring that can possibly be completed is completed. They then added this to the criteria for an economic set: if any topological ring has not been completed by their previous algorithm - as is the case in figure 5(c) - then a line that does complete it is added. The results of this improved algorithm, when applied to published hand-drawn examples from Hillier et al (1983), are so close to the originals as to be almost indistinguishable (see Peponis et al, 1998, page 572).

Peponis et al point out that there is a fundamental problem with greedy algorithms in general: they are not guaranteed to find the minimal set, though they may well do so in certain instances. This is, perhaps, academic; Peponis et al have defined $a$ rigorous method for their particular configurational analysis. It yields an economic set of lines that (in their own words) "get everywhere" and complete all circulation loops. 

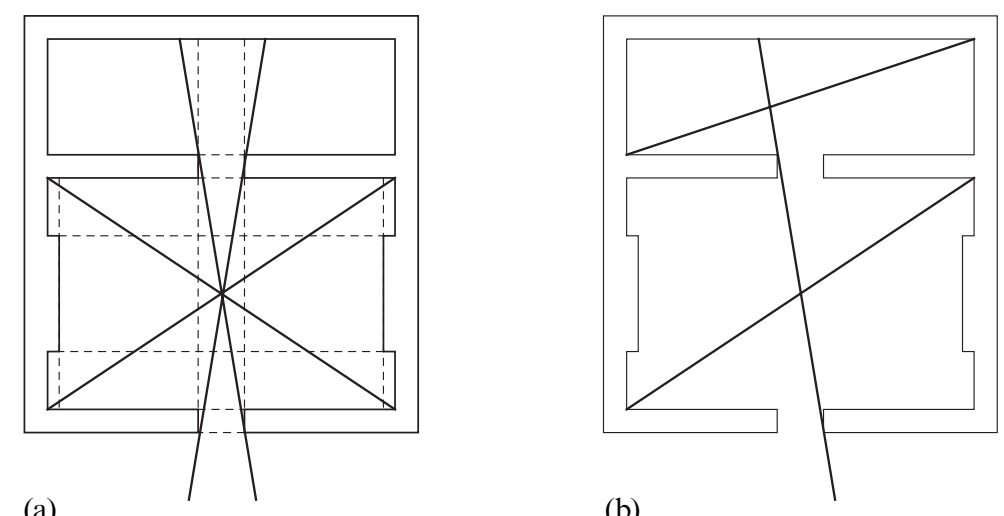

Figure 6. (a) Details on wall surfaces form a pathological case for Peponis et al's algorithm. m-lines are shown in bold, with doted s-lines sketched over. The alcoves to the left and right overproduce axial lines (or m-lines) in the midsection, whereas, the corridor to the top is only passed through weakly. (b) An 'ideal' coverage of the system.

Although in most cases it will produce a unique set of axial lines for any particular configuration, this is not guaranteed. If two lines cross identical numbers of s-lines, Peponis et al choose to keep the longest; if they are both the same length, they choose to keep either one at random. This could have a fundamental effect on the outcome if the die is cast early on in the procedure. However, in most real systems this would seem to be extremely improbable, as evidenced by Peponis et al's close algorithmic reproductions of hand-drawn maps. So, if their results are so good, why attempt to redefine axial maps again?

Our answer is twofold: for practical and theoretical reasons. First, on practical grounds, although Peponis et al's method reproduces the original hand-drawings well, and better than we do herein, it requires that real cartographers' plans be simplified before the method can work. The condition that all s-lines must be cut is too strong for plans in general. The s-lines can be created by, for example, meanderings along a street, or the buttresses of a church - both of which are present in Hillier and Hanson's original map of Gassin shown in figure 1. If these are included, then thousands of lines are necessary to cross absolutely all s-lines. Thus, scale of representation becomes a crucial concern. Figure 6 shows a simplified case to demonstrate the point: in the figure 'extra' axial lines are required to get into alcove spaces, which would not automatically be drawn by a person. Rather than simplify plans as Peponis et al do, we show herein that it is possible to generate a good axial map from the base data themselves. Second, on theoretical grounds, for these lines to form an axial map, we believe their definition needs an adjustment. We believe that the connectedness of the graph should be primary because it completes the final phrase of Hillier and Hanson's (1984) definition: that all axial links should be made. Although Peponis et al imply an attempt to maximise connectedness while minimising number of lines-longest lines are chosen in s-line cutting ties because this maximises the chance that they will connect with other lines (1998, page 566) - the method is not guaranteed to make all axial links. Herein, we show how another simple formal minimisation technique can lead to a map which is better connected.

However, there is third, also theoretical, issue, demonstrated in figure 6, which we are unable to resolve. The corridor to the top is seemingly not fully 'covered'. Hillier and Penn (2004) call this corridor coverage as it stands 'weak', as it crosses the room across its width. The ideal coverage, they suggest, would somehow cut the space 
associated with the room lengthways, 'strongly', that is, along the longest axis of the convex space associated with the points in the room, as shown in figure 6(b).

\subsection{Isovist analysis retrieval}

Batty and Rana (2004) propose a similar solution to that of Peponis et al (1998). They too first simplify the problem by generating a candidate set of lines and then reduce this set by using a greedy algorithm. However, rather than starting with a geometric plan of a polygon with holes, they pixelise a map of the open space of the system to be considered. This has the advantage that it is free from scaling concerns after the pixel size has been decided, and nonpolygonal boundaries, such as curves or fractal surfaces, may be used. They then make a simplification akin to the selection of an all-line map: for every pixel in their map they construct an isovist (or viewshed) for the location (Benedikt, 1979). Batty and Rana note that the diagonals of the isovists (the longest lines through the centre point) correspond to the axial lines; hence, they generate a set of candidate lines from the sampled system. They then reduce this candidate set by using a greedy algorithm to approximate a solution to the art gallery guard-placement problem [to which a covering set of isovists corresponds (Davis and Benedikt, 1979)]. They choose a 'dominant' isovist according to some criterion-not necessarily longest diagonal [which would recreate Peponis et al's (1998) preliminary results], but also other isovist properties such as circularity and average radius (Benedikt, 1979; Batty, 2001). As it works simply on surveillability, the method will, in general, fail to complete all circulation loops (that is, topological rings - see above), but it does allow researchers to investigate a range of economic set generation methods. Batty and Rana (2004) note themselves that their "methods generate different results from those which were originally derived manually and intuitively" (page 617), and their results (page 631) show that many of the maps are quite dissimilar to those of Hillier and Hanson; this dissimilarity is reflected in their measurements of the systems. To Batty and Rana this is not a problem, as they are more concerned with a thorough investigation of the range of various axial-like maps that may be produced through simple application of rules. In addition to that of Batty and Rana, a second method based on isovist analysis has been proposed by Carvalho and Batty (2003). Rather than take axial lines directly from a generated set, they produce a raster image of isovist diagonal lengths as plotted on the plan. The images have maximal 'ridges' where the diagonal length maxima chain together along street segments (and, usefully for providing strong coverage also along the longest diagonals of convex polygons). Carvalho and Batty apply a Hough transform to detect the ridge peaks and convert them to straight vector lines (for details see Illingworth and Kittler, 1988). The results is elegant and axial-like. However, as with Batty and Rana's (2004) results, Carvalho and Batty's resultant axial lines are not maps of the connecting 'stringy' space, and leave large holes of space unconnected.

\section{Method}

For our solution to the axial maps we take a slightly different approach to these methods: we first concentrate on the second half of the definition as we have stated it. This allows us to form a unique minimal axial graph of a system (although, as we will see, in exceptional circumstances, the lines which comprise the graph may differ) without recourse to a greedy algorithm. However, as with the previous methods of Barry and Rana (2004) and Penn et al (1998), we use a more restrictive definition of an axial line. Whether to generate the axial-line definition according to the all-line map or from a set of isovists is fairly arbitrary; we choose the all-line map on the basis that it does not require a decision on resolution of pixelation, and that it corresponds with how researchers draw axial lines: they move a ruler so that they find a longest line; 
this process will in general result in a line from the all-line set, although in some cases a longer line may align with the face of a polygon. We present our method in two parts. First, the reduction from the all-line map. Second, two of Peponis et al's (1998) conditions are implemented in order to ensure that a good axial map of the system is retrieved: ability to surveil the whole system (the first half of the definition), and the preservation of topological rings (a minor addition to the original definition). It should be noted that any reduction technique requires a minimisation algorithm of some sort, and the fact that we present the minimisation first does not mean that we are starting with it to the detriment of the conditions of surveillability and preservation of rings. Indeed, as we have discussed, the primary definition of the set of axial lines is one that surveils the entire system.

\subsection{Subset elimination}

As we have discussed, Hillier and Hanson (1984) define an axial map to include all 'axial links'. That is, it must include any axial line that will connect otherwiseunconnected lines together. In terms of a method that works by elimination, the condition is reversed: we cannot remove a line connecting two others if this would render those lines unconnected. We can encode this rule by using a subset-elimination algorithm. To do so, we write the connections for a line as a set, for example, as pictured in figure 7(a), $P$ has connections to $Q, R$, and $S$, so the set of connections $C_{P}$ for the line $P$ is $C_{P}=\{Q, R, S\}$. We then include the rule that if any line connects to a line that its neighbours do not then it is retained, otherwise it is removed. This rule can be encoded as follows: to each connection set, add the line itself, thus $C_{P}$ becomes $\{P, Q, R, S\}$ in the figure and, as another example, $C_{R}=\{P, Q, R\}$. Remove any lines whose connections form a subset of or equal to the connections for this line, and repeat until no more lines can be removed. This rule is shown more formally in pseudocode, for the set of axial lines $G$, as follows:

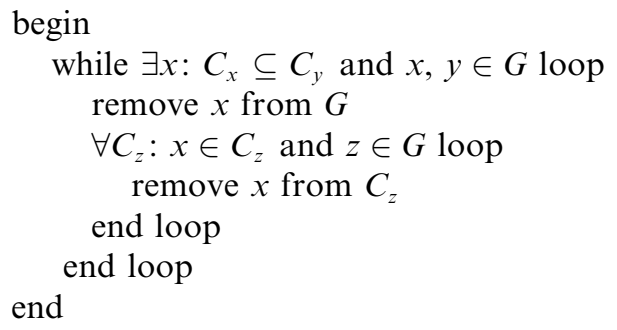

So, in figure 7(a), $Q$ is a subset of $P$ and it may be removed. However, the rule forces a choice upon us in the case of equal subsets. In fact, $C_{Q}=C_{R}$, so which do we remove?

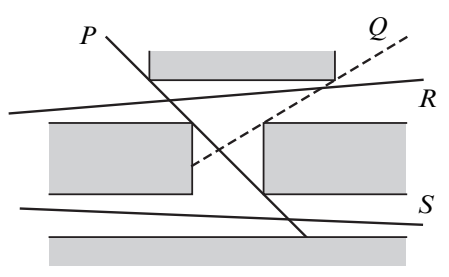

(a)

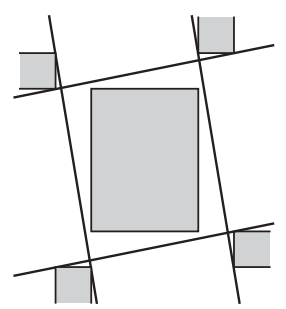

(b)

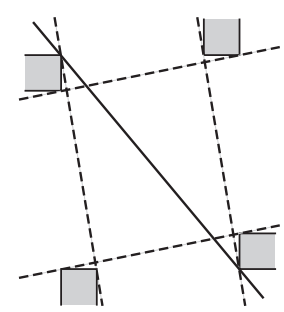

(c)

Figure 7. (a) Subset elimination preserves lines that connect otherwise-unconnected lines, for example, in this example, $P$ is retained rather than $Q$, as it connects to $S$. (b) Rings of lines 'lock' as each line has a connection which its neighbours do not. (c) Rings cannot be supported across open space, as the ring of lines is subsumed by a longer line that crosses them all. 
We choose to remove the shorter line or, if they are of equal length, either line. In figure 7(a), this means that $Q$ is removed in preference to $R$, even though they have the same connection set $C_{Q}=C_{R}=\{P, Q, R\}$. The reader will already have realised two things. First, that this appears to be a greedy algorithm, and second that this operation is a quick way of removing all lines from the all-line map set [for example, in figure 7(a) $Q$ then $R$ then $P$ then $S$ ].

Dealing with each in turn. This is not a greedy algorithm. The remaining graph is unique, whichever line we start with, no matter what initial set, as the stopping condition is that there does not exist a set that is a subset of or equal to any other. The 'choices' (of line length and other equally connected lines) are irrelevant to the graph as the connections are maintained. Variations in which lines are chosen can occur: for example, in figure 7(a), as we have discussed, we could remove $Q$ or $R$, but this does not affect the generality of our result for the overall process as resultant graphs are the same in terms of size and connectivity. The unique graph itself contains the minimum possible number of lines that can fulfil subset elimination, and no more.

The second concern is true only for systems without large circulation loops. If loops are composed of four or more lines, then the removal is halted at some point because the four lines 'lock' tight, as shown in figure 7(b). Each line is connected to two others, but not to the fourth, so the set of connections for each line is different. Note that this locking, though, can occur only around geometry. If there is a group of four lines across open space, there is necessarily a longer line which intersects them all, as shown in figure 7(c). What is more, the condition for subset elimination leads naturally to a primary facet of all hand-drawn maps: the fact that all the lines within the system remain connected into a single graph - a facet that other authors have either had to code for explicitly, or to deliberately ignore. Thus, when we look at the results of subset elimination (figure 8) we see a remarkable thing: the axial lines are almost what we would expect to have drawn by hand. The overlapping triangular rings around polygons are a feature of the elimination process, which we discuss below in more detail. In each of the overlapping rings, one line joins to another which its neighbours do not, making two different circulation rings in each case. It is current thinking in space syntax that these rings should be completed, as the choice of lines that can be drawn around a circular block tends to be manifold (but, because of the context, not infinite). However, in addition to the rings, the axial maps produced by this algorithm do contain real errors which we correct in the following sections. On closer inspection of the results, we see two things: that both surveillance and topological rings may, occasionally, be missing — as shown in figure 9. Thus, we introduce two necessary conditions that must be fulfilled before line removal can take place.

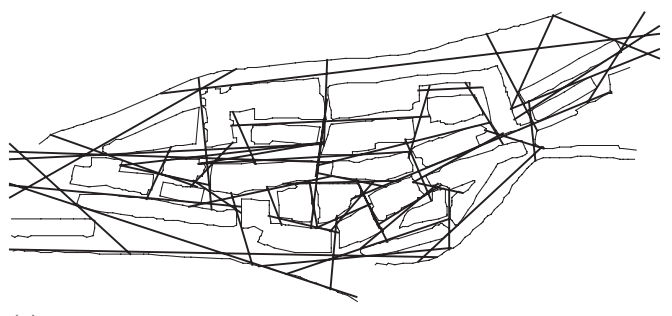

(a)

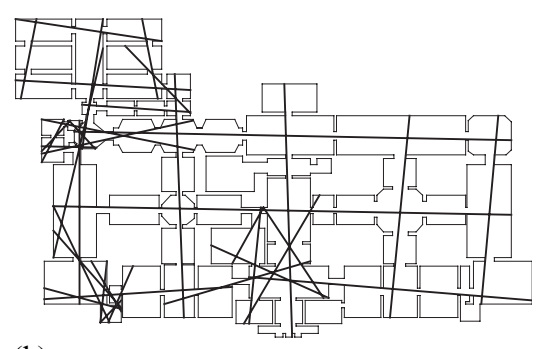

(b)

Figure 8. Demonstration of subset elimination (a) for the map of Gassin (b) for a simplified plan of the National Gallery, London. 


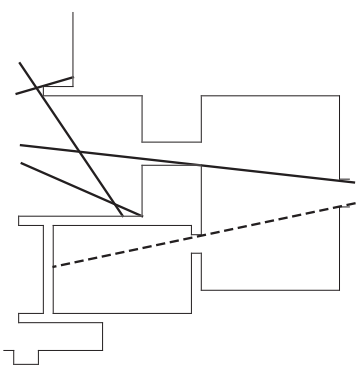

(a)

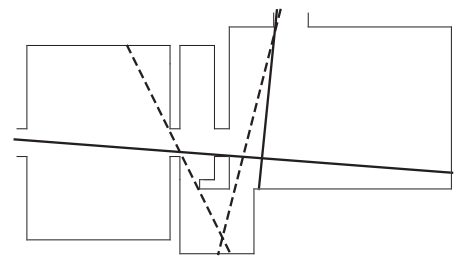

(b)

Figure 9. Detail of the National Gallery subset-elimination map: (a) not all locations are surveilled; (b) not all topological rings are completed. The extra dotted lines need to be added. Note that both rules together, not one or other rule, are necessary to complete a true axial map.

\subsection{Necessary conditions}

We now supply two necessary conditions which augment the subset-elimination algorithm. In each case, the elimination algorithm is forbidden from removing a line if it is vital either to surveil the whole space or to complete a topological ring.

\subsubsection{Surveillance}

In order to surveil the entire space, every point must be visible from at least one line. We can simplify this to say that every vertex on polygon boundaries must be visible from a line, as if any pair of adjacent vertices are visible, then the entire edge that conjoins them must also be visible. We can form a sufficient set of lines to fulfil this condition in an elegant fashion. To avoid confusion, we will start by calling the original all-line map the generation set of lines, $G$. Now we can form the set of axial lines from the generation set that passes through a particular point $p, G(p)$. In order for the point $p$ to be surveilled from at least one line in the reduced axial map, $R$, at least one of the lines in its generation set $G(p)$ must be crossed, as shown in figure 10(a). It should be noted at this point that this will not produce the minimal set of lines, as the line in $R$ only needs to cross at least one ${ }^{(3)} \mathrm{s}$-line associated with the point $p$ - as shown in figure 10(b). However, as discussed in section 2.1, we know that it is NP-hard to form the minimal set of lines that completely surveil the system; therefore, this approximation strikes us as a simple and effective method of retaining an economic set using lines that are already generated.

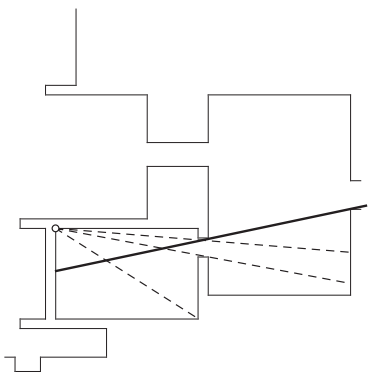

(a)

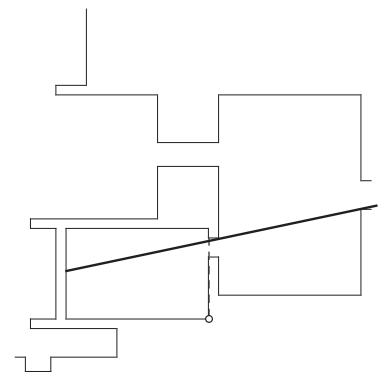

(b)

Figure 10. (a) A point is visible if at least one of its generated lines is crossed by an axial line. (b) Technically, for a minimal surveillance set, a line only needs to cross at least one s-line (or an axial line) for a point to remain visible.

(3) Although this may sound like Peponis et al's (1998) algorithm, it is in fact a very different condition to require that at least one s-line or an axial line must be crossed for each point, rather than that all s-lines must be crossed. 


\subsubsection{Topological rings}

In addition to surveillance, we follow Hillier and Hanson's (1984) implication and Peponis et al's (1998) argument that all topological rings should be completed. Our algorithm is again simple. For each point $p$ on the polygon edges, we separate it from all faces of other polygons that are visible from it. With the same sort of logic as that used in section 3.2.1, if any pair of adjacent vertices is separated from all the faces they can see, then the edge that conjoins them is also separated from the faces. Therefore, if every point on a polygon $P$ is separated from the faces of all other polygons, all polygons are separated from each other by lines. Thus, all topological rings are completed. To effect this technique, we form the set of triangular axial partitions from each point $p$ for every polygon. That is, with the axial lines from the generation set $G$ we form triangular partitions for each pair of lines (and also axial stubs formed without reflex extensions) emanating from $p$, as shown in figure 11 . The condition we apply to ensure that topological rings are completed is that the reduced axial set $R$ must include a line that passes from one side to another of each axial partition in order to separate $p$ from the opposing edge, as shown in figure 11(a). ${ }^{(4)}$ There is a special case that must be accounted for, which is shown in figure 11(b): where two lines, each of which passes through a separate edge of the triangular axial partition, intersect within it, then both together may be considered sufficient to separate the opposing edge from $p$. Our algorithm checks that either the first or second of these conditions is true for all triangular axial partitions, and allows removal of a line only if either of them is satisfied by other lines in the reduced set. The algorithm is particularly efficient, as we have already had to calculate the line intersections between lines in $G$ in order to meet the conditions both of surveillance and of subset elimination. Figures 11(c) and 11(d) show cases that might otherwise be ambiguous: (c) a line may pass through a point $p$ and still separate it from an edge (although it must pass with the polygon faces adjoining the point $p$ on one side and the opposing polygon edge on the other in order to do so); and, (d) a triangular axial partition may extend outwards from reflex corners until it hits the edge.

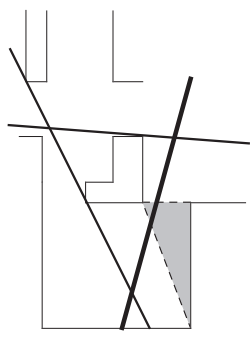

(a)

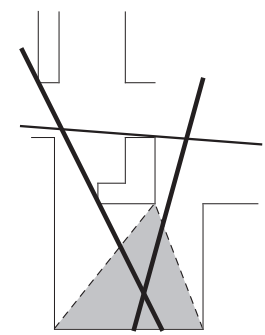

(b)

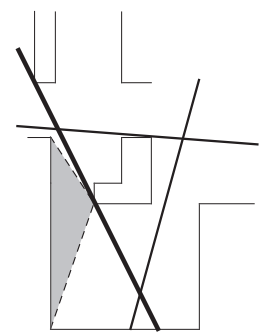

(c)

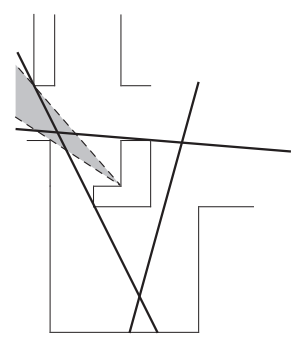

(d)

Figure 11. (a) A topologically sufficient set can be constructed on a vertex-by-vertex basis, by ensuring that lines pass from one side to the other through all the triangular axial partitions generated from it, or (b) that pairs of lines that pass through opposite sides cross within it. (c) Even if the line passes through a vertex, provided it separates the point from the opposing face, it is counted as part of the topologically sufficient set. (d) Note that triangular axial partitions extend out past reflex corners to any opposing polygon edge.

(4) The triangulation of space forms a solution to solve the art-gallery problem for polygons with holes: Bjorling-Sachs and Souvaine (1995) first derive triangular 'channels' to the polygon boundary before triangulating the separated polygons. They go on to show that guards placed at the vertices of these triangles form a sufficient set to surveil the entire space. 


\section{Discussion}

\subsection{Application}

The algorithm described in section 3 was encoded into University College London's Depthmap program, and applied to three example systems: a detailed map of Gassin vectorised from figure 28 in Hillier and Hanson (1984, page 91); a simplified plan of the National Gallery, London, drawn by the authors; and a further example taken from Hillier and Hanson (1984) - the urban area of Barnsbury in North London. (5) Both the original map of Gassin and that of Barnsbury in Hillier and Hanson (1984) have incomplete polygonal boundaries. Our approach to Barnsbury is described below; for Gassin, the map border was completed with rectangular sections such that all the lines on the original were included within the system. In all, the Gassin map has 1080 polygonal edges, and forms an all-line map with 5217 lines. Figure 12 shows the results of applying our algorithm to the systems. Although we would call the subset-elimination method 'correct' (we discuss why below, with reference to Barnsbury), we also used a greedy algorithm - applied to the remaining set of lines after subset elimination had been performed, and based on retaining maximum connectivity subject to surveillability and topological constraints - for comparison with the original map in Hillier and Hanson (1984) and Peponis et al's (1998) results. Table 1 (over) shows comparative measures of the resultant graphs for Gassin. For purists, it should be noted that the topological-rings condition makes no difference to the lines obtained by subset reduction (this is also true for Barnsbury, see below), and that this map therefore corresponds with the original definition of the axial map. The greedy algorithm demonstrates the effectiveness of our reduction method applied to Gassin. The graph has fewer lines, yet it is significantly better connected and better integrated [a measure of graph centrality introduced by Hillier and Hanson (1984)] than either the original or

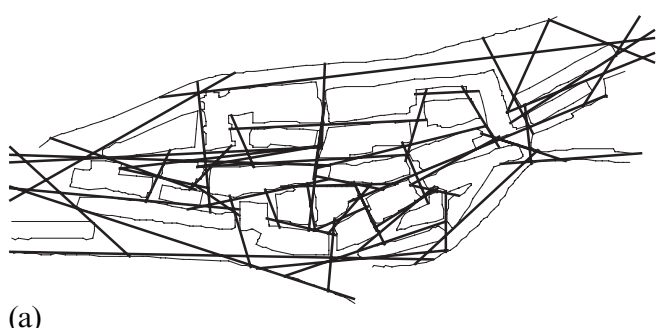

(a)

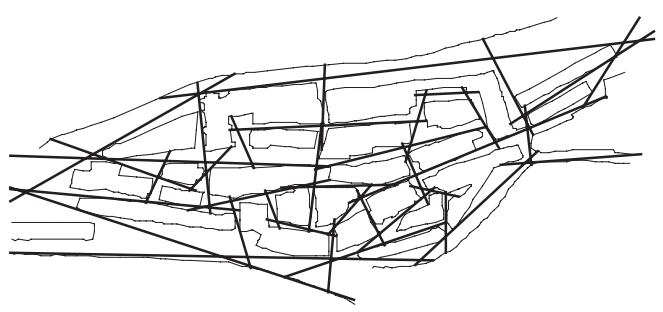

(c)

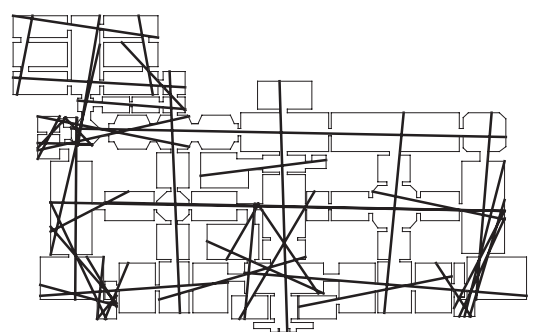

(b)

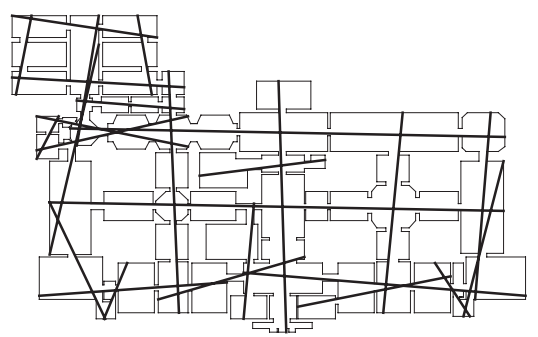

(d)

Figure 12. Preservation of surveillance and topological rings: (a) and (b) - by use of subset elimination; (c) and (d) - by use of a greedy algorithm.

(5) The software we utilise and vector drawings of the plans we use are available through University College London-see the section on supplementary information at the end of this paper for details. 
Table 1. Comparative measures of the different axial maps of Gassin.

\begin{tabular}{|c|c|c|c|c|c|}
\hline \multirow[t]{2}{*}{ Gassin map } & \multirow[t]{2}{*}{ Number of lines } & \multicolumn{2}{|c|}{ Connectivity } & \multicolumn{2}{|c|}{ Integration } \\
\hline & & average & $\mathrm{sd}^{\mathrm{a}}$ & average & sd \\
\hline All-line map & 5217 & 269.7 & 153.6 & 4.47 & 0.62 \\
\hline Subset elimination & 58 & 7.0 & 2.6 & 2.04 & 0.34 \\
\hline Greedy algorithm & 38 & 4.4 & 1.6 & 1.68 & 0.29 \\
\hline Peponis et $\mathrm{al}^{\mathrm{b}}$ & 39 & 4.0 & & 1.51 & \\
\hline Hillier and Hanson (1984) & 41 & 4.0 & 1.4 & 1.50 & 0.28 \\
\hline
\end{tabular}

Peponis et al's example. The algorithm achieves better connectivity by what are often quite subtle adjustments of the lines so that they just manage to connect to an extra line. This, or course, fulfils Hillier and Hanson's condition that all axial links should be made where they can be made. There are good reasons for the discrepancy with the original. Hillier and Hanson point out that they take into account topography of the landscape when drawing the lines; Gassin is a hill village, and so staircases join various parts of the town. In places, Hillier and Hanson appear to model these breaks in homogeneity by inserting two axial lines. There are also possible reasons for discrepancy with Peponis et al's results: first, because their algorithm does not optimise connectivity, it is simply missing the opportunity in order to optimise another variable; second, as a simplified plan must be used in Peponis et al's method, perhaps some lines do not quite pass through gaps that exist in the more accurate plan we use.

As well as analysis of Gassin and the National Gallery, we also analysed another example published by Hillier and Hanson - the area of Barnsbury in North London. This example serves to highlight the issues of subjectivity of cartography which must be considered, and also the subset-elimination feature of depth minimisation. The problem with cartography becomes apparent when tracing the map of open space, from Hillier and Hanson (1984, page 124, figure 62). The axial map they draw relates to the publicly accessible open space, yet the Ordnance Survey map they reprint includes both publicly accessible and inaccessible space. Not only this: Hillier and Hanson also include some intermediate, semipublic, space as dotted axial lines. On the map, not all the features they include are visible. Batty and Rana (2004) point out that this is a crucial difficulty with representations of space in general. In this case, we suggest, as we have in the past (Turner et al, 2001), that the crucial factor is the use to which the map is to be put. If the map is for understanding the interaction of society and space at the level of the individual, then the map must be to the scale of the individual. The individual's choices within space tend to be concerned with the major features of the landscape: roads are followed but trees are largely ignored and merely avoided, thus the level of detail of the road would seem appropriate. Unfortunately, the judge of the appropriate scale will always be human. For the Barnsbury area we take the openly public space, although even for public space some decision needs to be taken on whether or not to include certain back alleys and not others. The outer border of the map is also debatable, as it is not included by Hillier and Hanson. We followed a contemporary Ordnance Survey map to add a polygonal border to the region which encompassed their original map, to finish with the map shown in figure 13(a). Hillier and Hanson's axial map is shown in figure 13(b), and the results of our algorithm are shown in figures 13(c) and (d); a comparison of the graph measures is shown in table 2. 


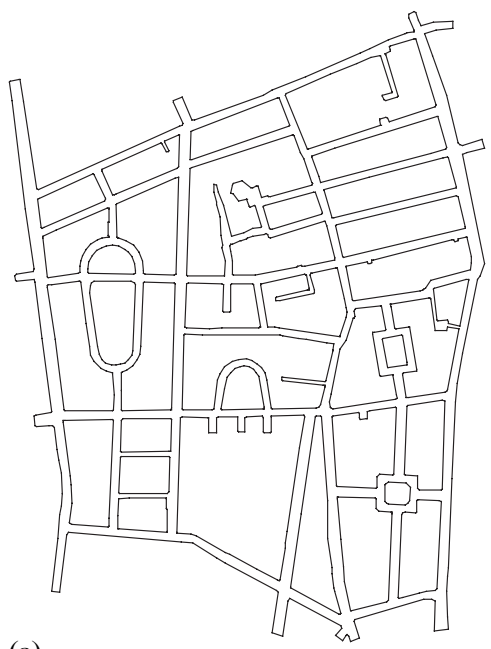

(a)

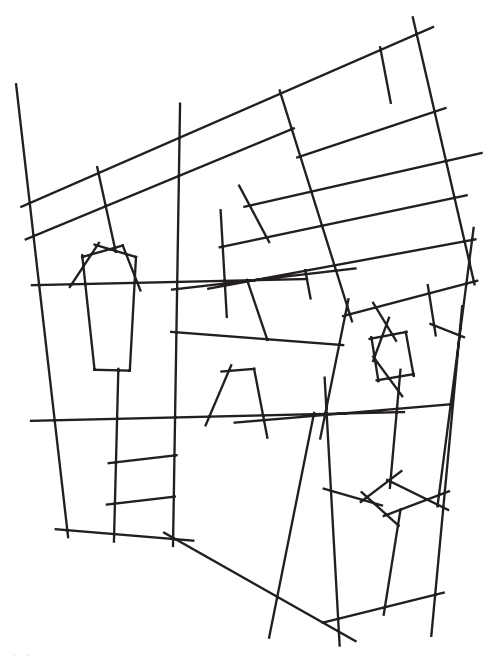

(c)

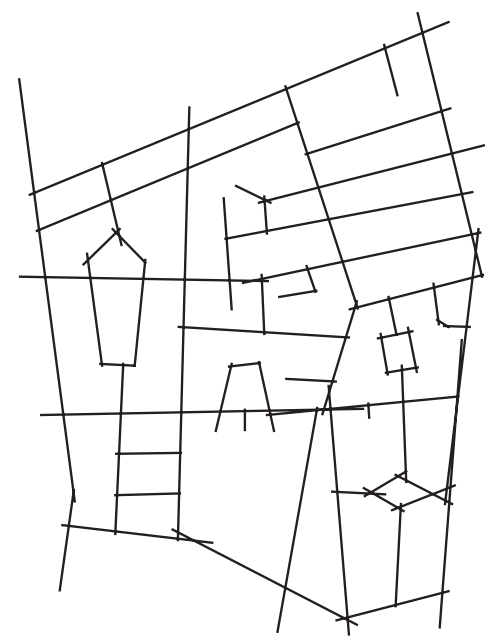

(b)

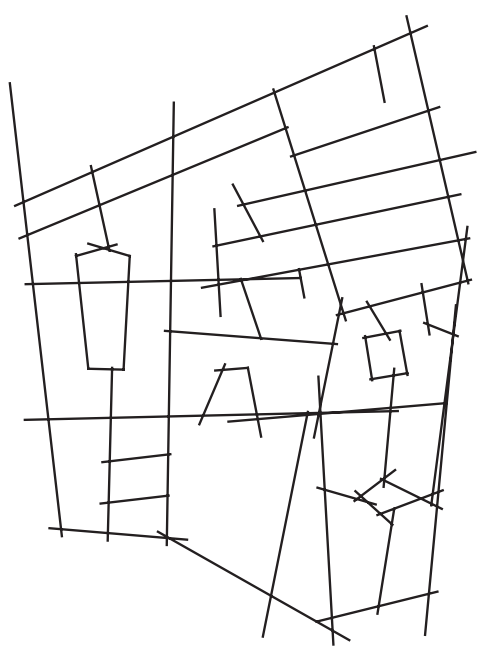

(d)

Figure 13. (a) Map of the publicly accessible space in Barnsbury (after Hillier and Hanson, 1984, page 124, figure 62). (b) The original axial map (after the axial map shown in Hillier and Hanson, 1984, page 125, figure 63). (c) The axial map as generated by subset elimination. (d) Axial map generated by using a greedy algorithm.

Table 2. Comparative measures of the different axial maps of Barnsbury.

\begin{tabular}{|c|c|c|c|c|c|}
\hline \multirow[t]{2}{*}{ Barnsbury map } & \multirow[t]{2}{*}{ Number of lines } & \multicolumn{2}{|c|}{ Connectivity } & \multicolumn{2}{|c|}{ Integration } \\
\hline & & average & $\mathrm{sd}^{\mathrm{a}}$ & average & sd \\
\hline All-line map & 1864 & 112.8 & 78.0 & 10.32 & 2.53 \\
\hline Subset elimination & 59 & 4.5 & 2.2 & 5.26 & 1.24 \\
\hline Greedy algorithm & 54 & 4.1 & 2.1 & 5.23 & 1.26 \\
\hline Hillier and Hanson (1984) & 61 & 3.8 & 2.2 & 4.97 & 1.22 \\
\hline${ }^{\mathrm{a}} \mathrm{sd}$-standard deviation & & & & & \\
\hline
\end{tabular}


In addition to the problems of scale of representation, our analysis of Barnsbury also highlights the feature of depth minimisation: that is, Hillier and Hanson's (1984) original statement that all axial links should be made. The result of filling in these links is to minimise the number of steps, or path length, or depth between axial lines in the graph, as figure 14(a) shows. Because Hillier and Hanson demand it, students of space syntax are told to perform this depth minimisation; however, as can be seen from the original in figure 14(b), Hillier and Hanson did not originally draw the depth-minimising lines - they preferred fewer lines over depth minimisation. This inconsistency has caused a dilemma not only for the student, but also for the tutor. A later version of the Barnsbury axial map presented by Hillier (1999) contains a diamond round this square, rather than the group of four lines in the 1984 version [compare figures 14(b) and 14(c)]. We should note that this is only one minor difference after fifteen years: most of the lines of the map are exactly the same. A pedantic note is that it is not technically possible to fit a diamond shape round the square in the map - it is slightly asymmetric $^{(6)}$ - but the results of Hillier's (1999) attempt to do so result from the struggle to minimise depth versus the struggle to represent the space with the fewest lines. Our algorithm reveals the answer is that a 'correct' map, as discovered by subset elimination, would incorporate both the depth-minimising lines and the pedantic route around the block.

\subsection{Time efficiency}

Although it is always difficult to compare how fast different algorithms work, given the range of different systems, it is worth noting the time efficiency of the algorithms. We recorded the length of time needed to process the different systems, using the standard Depthmap program on a PC with an Athlon XP2400+ chip running at $2 \mathrm{GHz}$. The results are shown in table 3; the time quoted for stage two includes both subset elimination and greedy algorithm. It should be noted that our implementation within Depthmap is only a proof of concept, so it is quite possible that considerable improvement could be made to these times. Most of the processing time shown in the table was used to construct the topological testing set (about $95 \%$ of the time for stage 2), although the complexity of the algorithm is only dependent on connectivity of the generation set $G$ [the algorithm is of the order $\mathrm{O}(n k)$ where $k$ is the average connectivity of $G$; in general, $k \ll n$ ]. Subset elimination is itself computationally fast as it does not require much sorting, and our algorithm approaches $\mathrm{O}(n \ln n)$ time for this phase of the algorithm. Although it does not appear so from these figures, the current implementation is most limited by the all-line map creation - which has simply been coded to work in $\mathrm{O}\left(n^{2}\right)$ time (check whether each polygon vertex can see each other polygon vertex). Thus, complex vector maps with many vertices (such as land-line data from Ordnance Survey) will take much longer. We intend to replace this algorithm with an $\mathrm{O}(n k)$ algorithm in the near future.

Table 3. Approximate time required to generate axial maps, in seconds.

\begin{tabular}{lccr}
\hline Area & $\begin{array}{l}\text { Stage 1: } \\
\text { all-line map }\end{array}$ & $\begin{array}{l}\text { Stage 2: } \\
\text { fewest-line map }\end{array}$ & Total \\
\hline Gassin & 15 & 104 & 119 \\
National Gallery & 3 & 9 & 12 \\
Barnsbury & 4 & 9 & 13
\end{tabular}

(6) Problems associated with scale are highlighted again. From the original map data, one can draw the depth-minimised route to the left of the square, but more recent and accurate map data [figure 14(d)] show that it is actually possible only if the front gardens of the houses are excluded. 

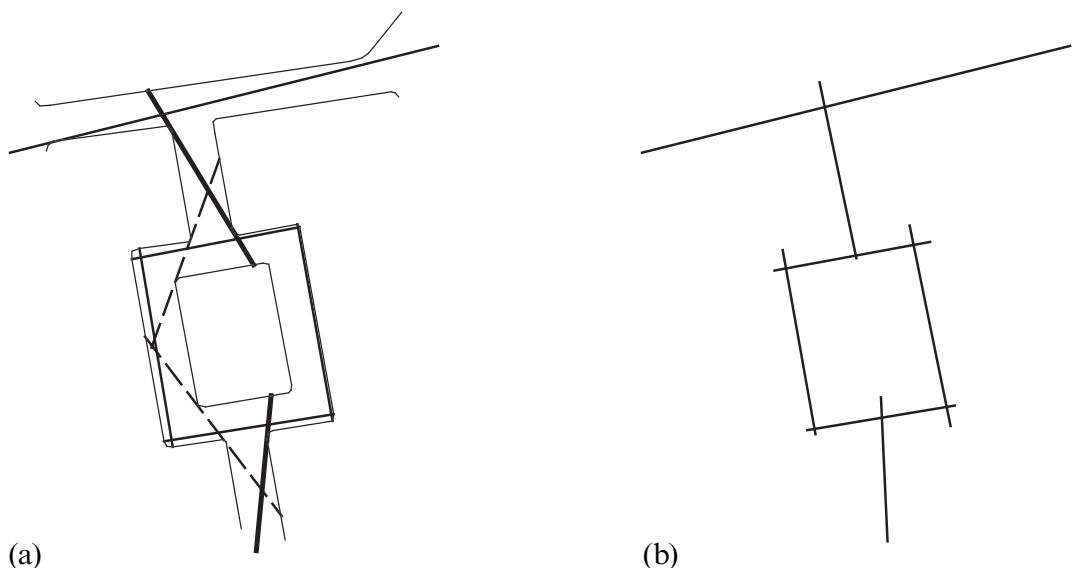

(b)
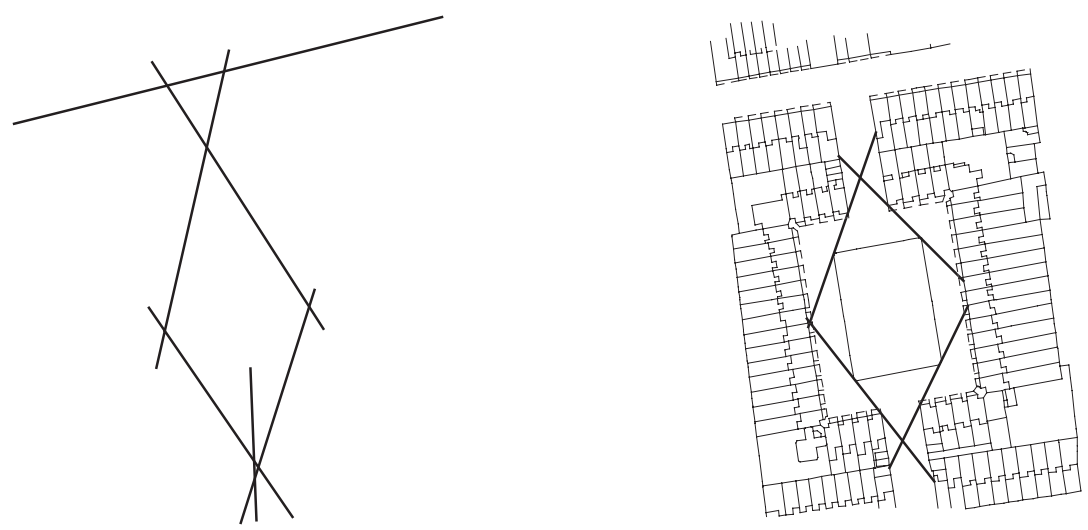

(c)

(d)

Figure 14. (a) Detail of the subset-elimination axial map of Barnsbury. Notice how the dotted lines minimise the depth between the lines drawn in bold. (b) Detail of the map as drawn by Hillier and Hanson (1984, page 125, figure 63). (c) Detail of the map as drawn by Hillier (1999, page 113, figure 5). (d) Detail of contemporary Ordnance Survey land-line data, (C) Crown Copyright, used with kind permission. The route to the left may be completed if front gardens (shown with dotted lines) are excluded. The route to the right is not completable.

\section{Conclusion}

We have demonstrated a method to retrieve axial lines algorithmically from any given plan specified in terms of a polygon with holes, that is, a typical plan of a building or map of an urban area. Our algorithm draws heavily on Peponis et al's (1998) method of axial-map construction. In particular, we start with an all-line map of the system (a set of lines generated from the vertices of the building and boundary polygons, which is constructed from lines extended from intervisible vertices within the space of the plan). Also following Peponis et al, we apply a process that reduces this set to an economical set of lines that both surveils the entire system, and also partitions the space into topological rings. We make two enhancements to Peponis et al's technique. First, we provide an algorithm that works from an axial graph, that is, a graph constructed by the use of axial lines as nodes and their crossing points as connections. From this we are able to fulfil Hillier and Hanson's (1984) original condition of an axial map: that it should make all possible connections between lines that are not otherwise connected. 
In doing so, we realise that a unique minimal axial graph is achievable through subset elimination of axial lines from the graph. Second, our algorithm is better suited to multifaceted polygons as found in generic map data. As the only input is vector line data, we hope to apply the system to the production of axial maps for urban areas from Ordnance Survey land-line data in the near future, and thus to automate fully the procedure of axial-map generation.

This demonstration highlights one further property of the axial map: that the map representation is derivable directly from the way vertices on built-form boundaries relate to each other through open space. The axial graph therefore provides a highly economic representation of intervisibility from which approximations may be derived of minimum direction change and minimum travel distance through open space in a built environment. It is perhaps these properties that account in part for the explanatory power of the axial representation in the extensive empirical research in which space-syntax methods are used.

In the course of this research, we have found, like Peponis et al before us, that the key elements to describe the 'stringiness' and to 'cover completely' a space are the surveillability of the entire system and the topology of the space. To a major extent, it appears these are the rules people are following when they draw axial systems. However, there is one aspect of the coverage drawn by people that neither we nor Peponis et al have managed to answer, and thus we finish with a challenge to the reader. In section 2.2 we discussed the problem of weak and strong coverage. 'Strong' coverage is the minimal set of axial lines that forms all axial links and crosses all maximal convex polygons along their longest axis. We showed a pathological case for Peponis et al's algorithm; the response of our algorithm is shown in figure 15(a). Although it does not overproduce lines, our method still leaves out the lines of strong coverage shown in figure 15(b). Our question is, how does one create this set of lines?

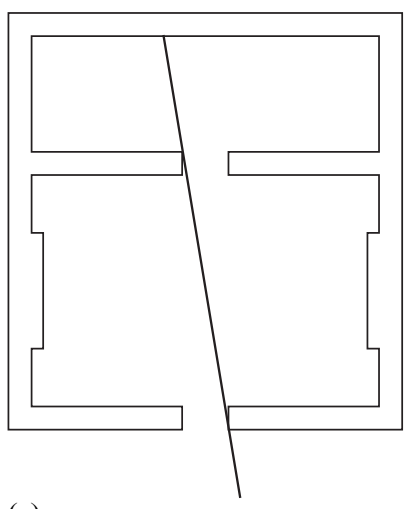

(a)

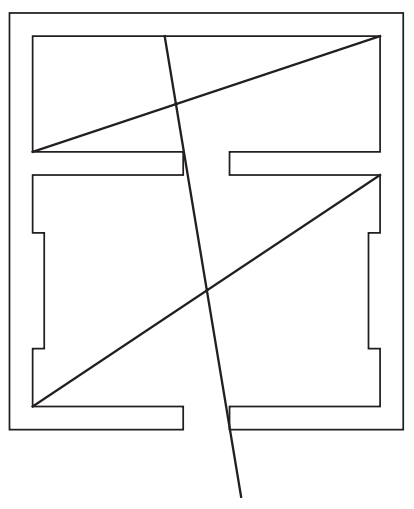

(b)

Figure 15. (a) The response of the algorithm to the pathological s-line case. (b) An 'ideal' coverage.

\section{Supplementary information}

We have DXF plans available for Gassin, the National Gallery, and Barnsbury, and a CAT file (a format native to Depthmap) for the pathological s-line example. Gassin, the National Gallery, and the pathological case can be downloaded from: http:// www.vr.ucl.ac.uk/research/axial/. In addition, the site includes results as encapsulated postscript as well as graph-analysis data in plain text format for all the axial maps discussed. The version of Depthmap used to obtain the results shown in this paper 
was 4.07 r. The Depthmap program is available to academic researchers through Space Syntax Limited; for details, see: http://www.vr.ucl.ac.uk/depthmap/. The Barnsbury map is covered by Crown copyright, so interested parties are asked to contact the authors directly to obtain it with the permission of Ordnance Survey.

Acknowledgements. The work this paper describes was part funded by EPSRC platform funding (GR/N21376/01). We are also grateful to the anonymous reviewers for their detailed comments.

\section{References}

Batty M, 2001, "Exploring isovist fields: space and shape in architectural and urban morphology" Environment and Planning B: Planning and Design 28123 - 150

Batty M, Rana S, 2004, "The automatic definition and generation of axial lines and axial maps" Environment and Planning B: Planning and Design 31 615-640

Benedikt M L, 1979, “To take hold of space: isovists and isovist fields" Environment and Planning B 6 $47-65$

Bjorling-Sachs I, Souvaine D L, 1995, "An efficient algorithm for guard placement in polygons with holes" Discrete Computational Geometry 1377 - 109

Carvalho R, Batty M, 2003, "A rigorous definition of axial lines: ridges on isovist fields", WP 69, Centre for Advanced Spatial Analysis, University College London, London

Carvalho R, Penn A, 2004, "Scaling and universality in the micro-structure of urban space" Physica A $332539-547$

Cutini V, Petri M, Santucci A, 2004, "From axial maps to mark point parameters analysis - a GIS implemented method to automate configurational analysis", in Computational Science and its Applications-ICCSA 2004 (Springer, Berlin) pp 1107-1116

Davis L S, Benedikt M L, 1979, "Computational models of space: isovists and isovist fields" Computer Graphics and Image Processing 11(3) 49-72

de Holanda F (Ed.), 1999 Proceedings of the 2nd International Symposium on Space Syntax Universidade de Brasilia, Brasilia, Brazil

Desyllas J, 2000 The Relationship between Urban Street Configuration and Office Rent Patterns in Berlin PhD thesis, Bartlett School of Graduate Studies, University College London, London

Hanson J (Ed.), 2003 Proceedings of the 4th International Symposium on Space Syntax University College London, London

Hillier B, 1999, "Centrality as a process: accounting for attraction inequalities in deformed grids" Urban Design International 4(3) 107 - 127

Hillier B, Hanson J, 1984 The Social Logic of Space (Cambridge University Press, Cambridge)

Hillier B, Penn A, 2004, "Rejoinder to Carlo Ratti" Environment and Planning B: Planning and Design $31501-511$

Hillier B, Shu S, 2000, "Crime and urban layout: the need for evidence", in Secure Foundations: Key Issues in Crime Prevention and Community Safety Eds S Ballintyne, K Pease, V McLaren (Institute for Public Policy Research, London) pp 224-248

Hillier B, Hanson J, Peponis J, Hudson J, Burdett R, 1983, "Space syntax” Architects Journal 178(48) $67-75$

Hillier B, Penn A, Hanson J, Grajewski T, Xu J, 1993, "Natural movement: or, configuration and attraction in urban pedestrian movement" Environment and Planning B: Planning and Design $2029-66$

Hoffman F, Kaufmann M, Kriegel K, 1991, "The art gallery theorem for polygons with holes", in Proceedings of the 32nd Annual Symposium of Foundations of Computer Science (IEEE Computer Society Press, Los Alamitos, CA) pages 39-48

Illingworth J, Kittler J, 1988, "A survey of the Hough transform" Computer Vision Graphics and Image Processing 44(1) 87 - 116

Jiang B, Claramunt C, 2002, "Integration of space syntax into GIS: new perspectives for urban morphology" Transactions in GIS 6295 - 309

Krüger M J T, 1979, "An approach to built-form connectivity at an urban scale: system description and its representation" Environment and Planning B: Planning and Design 6 67-88

Lee D T, Lin A K, 1986, "Computational complexity of art gallery problems" IEEE Transactions on Information Theory 32 276-282

March L, Steadman P, 1971 The Geometry of Environment (Methuen, London)

O'Rourke J, 1987 Art Gallery Theorems and Algorithms (Oxford University Press, New York) 
Penn A, Conroy R, Dalton N, Dekker L, Mottram C, Turner A, 1997, "Intelligent architecture: new tools for the three dimensional analysis of space and built form", in Proceedings of the 1st International Symposium on Space Syntax University College London, London, pp 30.1-30.19

Penn A, Hillier B, Banister D, Xu J, 1998, "Configurational modelling of urban movement networks", in Travel Behaviour Research: Updating the State of Play Eds J D Ortuzar, D Henshar, S Jara-Diaz (Pergamon, Amsterdam) pp 399-362

Peponis J, Hadjinikolaov E, Livieratos C, Fatouros D A, 1989, “The spatial core of urban culture" Ekistics 56(334/335) $43-55$

Peponis J, Wineman J, Bafna S, Rashid M, Kim S H, 1998, "On the generation of linear representations of spatial configuration" Environment and Planning B: Planning and Design $25559-576$

Peponis J, Wineman J, Bafna S (Eds), 2001 Proceedings of the 3rd International Symposium on Space Syntax Georgia Institute of Technology, Atlanta, GA

Ratti C, 2004, "Space syntax: some inconsistencies" Environment and Planning B: Planning and Design $31487-499$

Space Syntax Limited, 2003, "Selected portfolio of projects", http://www.spacesyntax.com/portfolio/ projects-profile/Selected.htm

Thomas G, Donikian S, 2000, "Modelling virtual cities dedicated to behavioural animation" Computer Graphics Forum 19(3) C71 - C80

Turner A, Doxa M, O'Sullivan D, Penn A, 2001, "From isovists to visibility graphs: a methodology for the analysis of architectural space" Environment and Planning B: Planning and Design $28103-121$

UCL, 1997 Proceedings of the 1st International Symposium on Space Syntax University College London, London 